PHYSICAL REVIEW D 67, 089902(E) (2003)

\title{
Erratum: Computing radiation from Kerr black holes: \\ Generalization of the Sasaki-Nakamura equation \\ [Phys. Rev. D 62, 044029 (2000)]
}

Scott A. Hughes

(Received 4 March 2003; published 28 April 2003)

DOI: 10.1103/PhysRevD.67.089902

PACS number(s): 04.25.Nx, 99.10.Cd

The second line of Eq. (4.5) should read

$$
{ }_{s} U_{1}(r)=V_{T}+\frac{1}{\beta \Delta^{s}}\left[\left(2 \alpha+\beta^{\prime} \Delta^{s+1}\right)^{\prime}-\frac{\eta^{\prime}}{\eta}\left(\alpha+\beta^{\prime} \Delta^{s+1}\right)\right] .
$$

As published, the factor before the first square bracket is incorrectly specialized to $s=2$. Note that this will lead to dimensional inconsistencies. I am very grateful to Vitor Cardoso for bringing this error to my attention. 